\title{
Quantitative Electroencephalography in Patients With Depression and Epilepsy Spectrum Disorder and Its Correlation With Clinical Features of Depression
}

\author{
PS Biswas, D Ram, SK Munda
}

\begin{abstract}
Objectives: To determine the associations of epilepsy spectrum disorder (ESD) with brain insult and certain quantitative electroencephalographic (QEEG) and clinico-demographic parameters in patients with depression.

Methods: 21 right-handed patients aged 18 to 50 years with the diagnosis of depression and ESD (scored $\geq 70$ in Iowa Interview for Partial seizure-like symptoms) were compared with 21 patients with depression but without ESD (scored $<70$ ) and 21 normal subjects with $<3$ positive scores on the 12 -Item General Health Questionnaire. Their QEEG parameters such as power spectrum and coherence of five frequency bands in 11 regions were compared.

Results: Patients with ESD had more minor traumatic brain injury along with more severe and multiple depressive episodes. Patients with ESD had significantly higher betal power over all regions on the left scalp than did normal subjects. Patients with ESD had significantly higher beta2 power over the left central region than did patients with no ESD and normal subjects.

Conclusions: For patients with severe recurrent depression, clinicians should systematically check for episodic partial seizure-like phenomena, especially when QEEG shows electrical disorganisation in the left side in those with mild traumatic brain injury.
\end{abstract}

Key words: Brain injuries; Depression; Electroencephalography; Epilepsy; Seizures

Partha Sarathi Biswas, Department of Psychiatry, Govind Ballabh Pant Institute of Postgraduate Medical Education and Research (Maulana Azad Medical College), New Delhi, India

D Ram, Central Institute of Psychiatry, Kanke, Ranchi, India

SK Munda, Central Institute of Psychiatry, Kanke, Ranchi, India

Address for correspondence: Partha Sarathi Biswas, Department of Psychiatry, 6th Floor, Academic Block, Govind Ballabh Pant Institute of Postgraduate Medical Education and Research (Maulana Azad Medical College), New Delhi, India

Email:drparthas@rocketmail.com

Submitted: 4 April 2020; Accepted: 28 Apr 2021

\section{Introduction}

Multiple partial seizure-like (paroxysmal affective, sensory, and cognitive) symptoms have been reported in those with persistent dysphoria and emotional liability ${ }^{1}$ or in those with head injury, ${ }^{2}$ depression, ${ }^{3}$ schizophrenia, ${ }^{4}$ or significant dissociation, ${ }^{5}$ or in those with paroxysmal theta bursts on electroencephalography (EEG), ${ }^{6}$ or even in normal persons. ${ }^{5,7}$ They are regarded as manifesting an epilepsy spectrum disorder (ESD) analogous to schizophrenia spectrum disorder. ${ }^{1,5}$ Unlike patients with classic complex partial seizure, these patients experience symptoms in an unpredictable sequence and simply report too many episodic symptoms. They seldom display classic motor automatisms such as lip-smacking and carphologia. Their EEGs are typically interpreted as within normal limits or as abnormalities not clearly epileptiform in nature., 5 They have no episode of extended loss of contact with the environment. They are more likely to seek (or be brought to) medical treatment for mood complaints (eg, persistent dysphoria, ego-dystonic rage outbursts), concerns about 'going crazy', transitory cognitive problems (eg, memory gaps, brief confusional spells, frequent word-finding lapses), or complaints of atypical headache and cephalic pain rather than classic epileptic phenomena. ${ }^{5}$

Development of multiple partial seizure-like symptoms has been reported after traumatic brain injury, hypoxia, or high fever, ${ }^{5-8}$ all of which may induce a hyperexcitable state in localised brain tissue by lowering the seizure threshold. Patients with ESD after traumatic brain injury have frequent and unusual EEG abnormalities without common epileptic seizures.9-12 The role of the hippocampus in epilepsy and kindling helps establish the association between behavioural dysfunction characteristic of ESD and its underlying pathophysiology. Anticonvulsant therapy may be indicated in patients with depression who have an underlying convulsive tendency, because $>90 \%$ of patients with ESD respond well to anticonvulsant mood stabiliser treatment. ${ }^{3,13-15}$ Clinicians should be cautious when treating these patients with tricyclic antidepressants, because tricyclic antidepressants lower seizure threshold and thus may exacerbate symptoms. Quantitative EEG (QEEG) can demonstrate underlying convulsive tendency 
in terms of power spectrum and coherence. Thus, this study aimed to determine the associations of ESD with brain insult, certain QEEG patterns, and clinico-demographical parameters in patients with depression.

\section{Methods and Materials}

This cross-sectional observational study was conducted between October 2008 and October 2009 and was approved by the Institutional Protocol Review Board of Central Institute Psychiatry, Ranchi, India. Written informed consent was obtained from each participant. We recruited 21 righthanded patients aged 18 to 50 years who had a diagnosis of depression (single or recurrent episode or bipolar affective disorder currently in depression with or without psychotic symptoms based on the ICD-10 $\mathrm{DCR}^{16}$ ) and the presence of ESD (indicated by a score of $\geq 70$ in Iowa Interview for Partial Seizure-like Symptoms ${ }^{5}$ [IIPSS]). In addition, we recruited 21 patients with depression but without ESD (score of $<70$ in IIPSS) and 21 normal subjects with $<3$ positive scores on the 12-Item General Health Questionnaire. Participants of the three groups were matched with respect to age, sex, and handedness (based on the Handedness Preference Schedule). They underwent a detailed physical examination to rule out any major medical or neurological disease. Their socio-demographic and clinical data were collected. Severity of depression was assessed using the Beck Depression Inventory-II (BDI-II).

Subjects were excluded if they had a history of major medical and neurological disorder, co-morbid major psychiatric disorder, significant brain injury (based on clinical examination and laboratory investigations), electroconvulsive therapy in the past 6 months, or previous use of oral psychotropic drugs in past 4 weeks or depot antipsychotics in past 8 weeks. Nonetheless, individuals with a history of mild traumatic brain injury (MTBI) as defined by the American Academy of Neurology ${ }^{17}$ were included. MTBI is defined as injury to the head arising from blunt trauma or acceleration or deceleration forces with one or more of the following conditions: (1) transient confusion, disorientation, or impaired consciousness for $<15$ minutes; (2) dysfunction of memory around the time of injury for $<15$ minutes; (3) loss of consciousness lasting either brief (seconds) or prolonged (<30 minutes); (4) seizures acutely following injury to the head; (5) irritability, lethargy, or vomiting following head injury, especially among infants and very young children; and (6) headache, dizziness, irritability, fatigue, or poor concentration, especially among older children and adults.

Participants were instructed to lie down and rest with their eyes closed but in an alert state while EEG (30 minutes) was recorded in a sound-proof light-attenuated room. A 32-channel digital EEG was recorded from 25 referential electrodes (FP1, FP2, F3, F4, C3, C4, P3, P4, O1, O2, F7, F8, T3, T4, T5, T6, FZ, CZ, PZ, PG1, PG2, A1, A2, T1, and $\mathrm{T} 2$ ). Ag- $\mathrm{AgCl}$ electrodes were applied on the scalp using the international 10-20 system. ${ }^{18}$ Electrode-skin impedance was kept at $<5 \mathrm{k} \Omega$. The AC filter and a notch filter $(50 \mathrm{~Hz})$ were turned on while acquiring data. The EEG amplifiers had a bandpass from 0.1 to $120 \mathrm{~Hz}$ ( $3 \mathrm{~dB}$ points). Data were sampled at $200 \mathrm{~Hz}$ with 12-bit resolution. 17 channels of the EEG from 17 electrodes (F3, F4, C3, C4, P3, P4, O1, O2, F7, F8, T3, T4, T5, T6, FZ, CZ, and PZ) were used for computerised analysis. FP1, FP2, T1, and T2 were not included because of higher chances of contamination with artefacts. EEG was visually inspected by an experienced EEG reviewer (who were blinded to group allocation) to exclude drowsiness and artefacts such as eye movements, electrocardiography, and electromyography. The reviewer then extracted 1 minute of artefact-free data from each patient's recording. The length of each epoch was $1 \mathrm{~s}$. Analogue to digital conversion was performed with 16 bits, time constant $0.1 \mathrm{~s}$, and $70 \mathrm{~Hz}$ high-cut frequency. The digitalisation rate was 500 samples/s/channel.

A fast Fourier transformation was used to acquire the digital data, which were saved as ASCII files. Absolute power and coherence values of five frequency bands: delta (0.5-3.5 Hz), theta $(4-7.5 \mathrm{~Hz})$, alpha $(8-12.5 \mathrm{~Hz})$, slow beta (13-20 Hz), and fast beta $(20.5-30 \mathrm{~Hz})$ were calculated by MATLAB v 7.0 .

11 regions were identified for power spectrum analysis: left frontal (F3), right frontal (F4), left temporal (F7, T3, T5), right temporal (F8, T4, T6), left central (C3), right central $(\mathrm{C} 4)$, left parietal $(\mathrm{P} 3)$, right parietal $(\mathrm{P} 4)$, left occipital (O1), right occipital (O2), and midline (FZ, CZ, PZ). Regional score of power/coherence was calculated by averaging individual scores of all channels of that region.

The same referential montages used for power spectrum are preferred for coherence analysis. ${ }^{19}$ Coherence was calculated for 39 combinations ${ }^{19}$ consisting of 7 interhemispheric and 32 intra-hemispheric channel pairs. Again, 25 scalp regions were constructed; they were frontal (F4F3), temporal (F8-F7, T4-T3, T6-T5), central (C4-C3), parietal (P4-P3), occipital (O2-O1), and left fronto central (F3-C3), right fronto central (F4-C4), left fronto parietal (F3-P3), right ronto parietal (F4-P4), left fronto temporal (F3-F7, F3-T3, F3-T5), right fronto temporal (F4-F8, F4-T4, F4-T6), left fronto occipital (F3-O1), right fronto occipital (F4-O2), left centro parietal (C3-P3), right centro parietal (C4-P4), left centro temporal (C3-T3), righty centro temporal (C4-T4), left centro occipital (C3-O1), right centro occipital (C4-O2), left parieto temporal (P3-F7, P3-T3, P3-T5), right parieto temporal (P4-F8, P4-T4, P4-T6), left parieto occipital (P3-O1), right parieto occipital (P4-O2), left temporo occipital (F7-O1, T3-O1,T5-O1), and right temporo occipital (F8-O2, T4-O2, T6-O2).

The absolute power and coherence of five frequency bands: delta, theta, alpha, slow beta (beta1), and fast beta (beta2) were analysed. Distribution of data was checked using the Q-Q plot and Kolmogorov Smirnov test. Distribution of scores of both power spectrum and coherence was non-normal. Thus, scores of power spectrum were log transformed (to the base 10), and scores of coherence were transformed with Fisher's Z to achieve the Gaussian 
distribution. Between-group comparisons were made using multivariate analysis (MANOVA) and analysis of variance.$^{20}$ Correlations between variables were evaluated using the Spearman correlations and point bi-serial correlation. Predictors for the ESD were identified using multiple stepwise linear regression analysis. Exponential function of all means was calculated from the log power data. Fisher's inverse transformation was calculated for scores of coherence. A p value (two tailed) of $<0.05$ was considered statistically significant.

\section{Results}

Each group consisted of 10 men and 11 women. Most participants were married (74.6\%), living in rural areas (71.4\%), and living with nuclear families $(63.5 \%)$. The three

Table. Characteristics and clinical profile of depressed participants with or without epilepsy spectrum disorder (ESD) and normal controls

\begin{tabular}{|c|c|c|c|c|c|}
\hline Variables & $\begin{array}{l}\text { ESD group } \\
(\mathbf{n}=\mathbf{2 1})^{*}\end{array}$ & $\begin{array}{l}\text { Non-ESD group } \\
\qquad(\mathbf{n}=\mathbf{2 1})^{*}\end{array}$ & $\begin{array}{l}\text { Normal control } \\
\quad(\mathbf{n}=\mathbf{2 1})^{*}\end{array}$ & $\mathbf{t} / \chi^{2} / \mathbf{F}(\mathbf{d f})$ & p Value \\
\hline Age, y & $32.43 \pm 7.74$ & $33.76 \pm 9.32$ & $32.52 \pm 7.67$ & $0.169(2,60)$ & 0.845 \\
\hline Education, $\mathrm{y}$ & $9.95 \pm 5.12$ & $10.33 \pm 4.73$ & $10.19 \pm 5.02$ & $0.032(2,60)$ & 0.969 \\
\hline Sex & & & & $0.000(2)$ & 1.000 \\
\hline Male & $10(47.6)$ & $10(47.6)$ & $10(47.6)$ & & \\
\hline Female & $11(52.4)$ & $11(52.4)$ & $11(52.4)$ & & \\
\hline Domicile & & & & $3.267(2)$ & 0.195 \\
\hline Rural & $13(61.9)$ & $18(85.7)$ & $14(66.7)$ & & \\
\hline Urban & $8(38.1)$ & $3(14.3)$ & $7(33.3)$ & & \\
\hline Marital status & & & & $0.670(2)$ & 0.715 \\
\hline Single & $6(28.6)$ & $4(19.0)$ & $6(28.6)$ & & \\
\hline Married & $15(71.4)$ & $17(81.0)$ & $15(71.4)$ & & \\
\hline Family type & & & & $0.137(2)$ & 0.934 \\
\hline Nuclear & $13(61.9)$ & $13(61.9)$ & $14(66.7)$ & & \\
\hline Extended & $8(38.1)$ & $8(38.1)$ & $7(33.3)$ & & \\
\hline Occupation & & & & $0.220(2)$ & 0.896 \\
\hline Unemployed & $3(14.3)$ & $4(19.0)$ & $4(19.0)$ & & \\
\hline Employed & $18(85.7)$ & $17(81.0)$ & $17(81.0)$ & & \\
\hline Duration of current episode, $\mathrm{m}$ & $4.93 \pm 3.49$ & $4.79 \pm 3.18$ & & $0.138(40)$ & 0.891 \\
\hline No. of previous episodes & $1.48 \pm 1.63$ & $2.43 \pm 4.38$ & & $-0.934(40)$ & 0.356 \\
\hline No. of total depressive episodes & $2.38 \pm 1.66$ & $2.43 \pm 3.12$ & & $-0.062(40)$ & 0.951 \\
\hline $\begin{array}{l}\text { Beck Depression Inventory-II } \\
\text { score }\end{array}$ & $41.71 \pm 5.14$ & $35.81 \pm 8.23$ & & $2.79(40)$ & 0.008 \\
\hline $\begin{array}{l}\text { Iowa Interview for Partial } \\
\text { Seizure-like Symptoms score }\end{array}$ & $76.05 \pm 5.33$ & $46.00 \pm 12.15$ & $14.57 \pm 7.49$ & $256.38(2,60)$ & $<0.001$ \\
\hline Depression type & & & & $0.42(2)$ & 0.846 \\
\hline Bipolar depression & $3(14.3)$ & $4(9.0)$ & & & \\
\hline Single episode depression & $8(38.1)$ & $9(42.9)$ & & & \\
\hline Recurrent depression & $10(47.6)$ & $8(38.1)$ & & & \\
\hline Brain insult & & & & $11.00(2)$ & 0.004 \\
\hline Present & $12(57.1)$ & $4(19.0)$ & $3(14.3)$ & & \\
\hline Absent & $9(42.9)$ & $17(81.0)$ & $18(85.7)$ & & \\
\hline Type of brain insult & & & & $12.46(6)$ & 0.019 \\
\hline Minor traumatic brain injury & $9(42.9)$ & $3(14.3)$ & $2(9.5)$ & & \\
\hline Anoxic spell & $1(4.8)$ & $1(4.8)$ & 0 & & \\
\hline High fever & $2(9.5)$ & 0 & $1(4.8)$ & & \\
\hline None & $9(42.9)$ & $17(81.0)$ & $18(85.7)$ & & \\
\hline
\end{tabular}

Data are presented as mean \pm standard deviation or No. (\%) 
groups were comparable in terms of age, sex, education level, domicile, marital status, family type, and occupation (Table).

Depression severity (BDI-II score) was higher in the ESD group than in the non-ESD group (41.71 vs 35.81, $\mathrm{p}=$ 0.008 , Table); $76.2 \%$ of patients in both groups had severe depression (BDI-II score of $>28$ ). The mean IIPSS score was highest in the ESD group, followed by the non-ESD and normal groups (76.05 vs 46.00 vs 14.57 , $\mathrm{p}<0.001$, Table). The most common depression type was recurrent depression (47.6\%) in the ESD group and single-episode depression (42.9\%) in the non-ESD group. $30.16 \%$ of all participants had brain insults, most frequently in the ESD group than in the non-ESD group or normal group $(57.1 \%$ vs $19.0 \%$ vs $14.3 \%, \chi^{2}=11.00, \mathrm{df}=2, \mathrm{p}=0.004$, Table). MTBI was most frequent in the ESD group than in the non-ESD group or normal group (42.9\% vs $14.3 \%$ vs $9.5 \%$, $\chi^{2}=12.46, \mathrm{df}=6, \mathrm{p}=0.019$, Table) .

The ESD group had significantly higher $(\mathrm{p}<0.05$, 2-tailed) beta1 power in the left-side frontal, temporal, central, parietal, and occipital regions than did the normal group [MANOVA: F (Pillai's Trace) $=1.327$, df $=10114$, $\mathrm{p}=0.224]$ but was comparable with the non-ESD group (Figure 1). In addition, the ESD group had significantly higher $(\mathrm{p}<0.05,2$-tailed) beta2 power in the left-side temporal and central regions than did the non-ESD group and the normal group [MANOVA: F (Pillai's Trace) $=1.458$, $\mathrm{df}=10114, \mathrm{p}=0.164$.], but post hoc test (with Bonferroni test) showed significantly higher beta 2 power only in the left-side central region (Figure 2). There was no significant

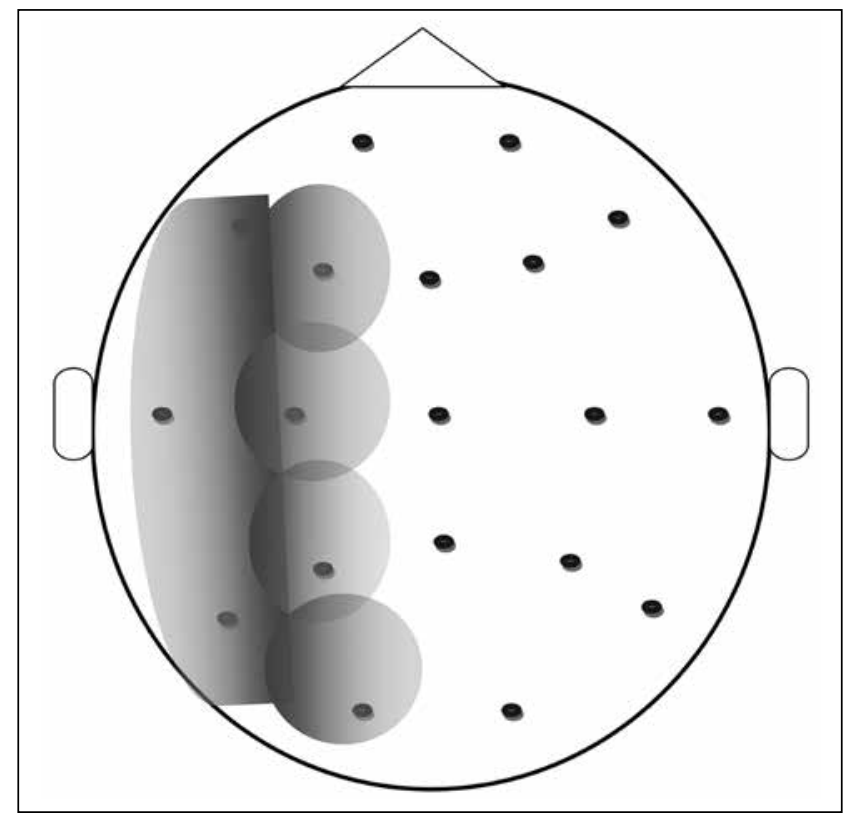

Figure 1. The epilepsy spectrum disorder (ESD) group has significantly higher betal power in the left-side frontal, temporal, central, parietal, and occipital regions than did the normal group but is comparable with the non-ESD group (effect size, medium $)^{20}$ group difference in spectral power in all five frequency bands in the right-side or midline region.

The duration of current episode negatively correlated with left temporal beta 2 power $(p=0.034$, Figure 3$)$. The IIPSS score positively correlated with the total number of

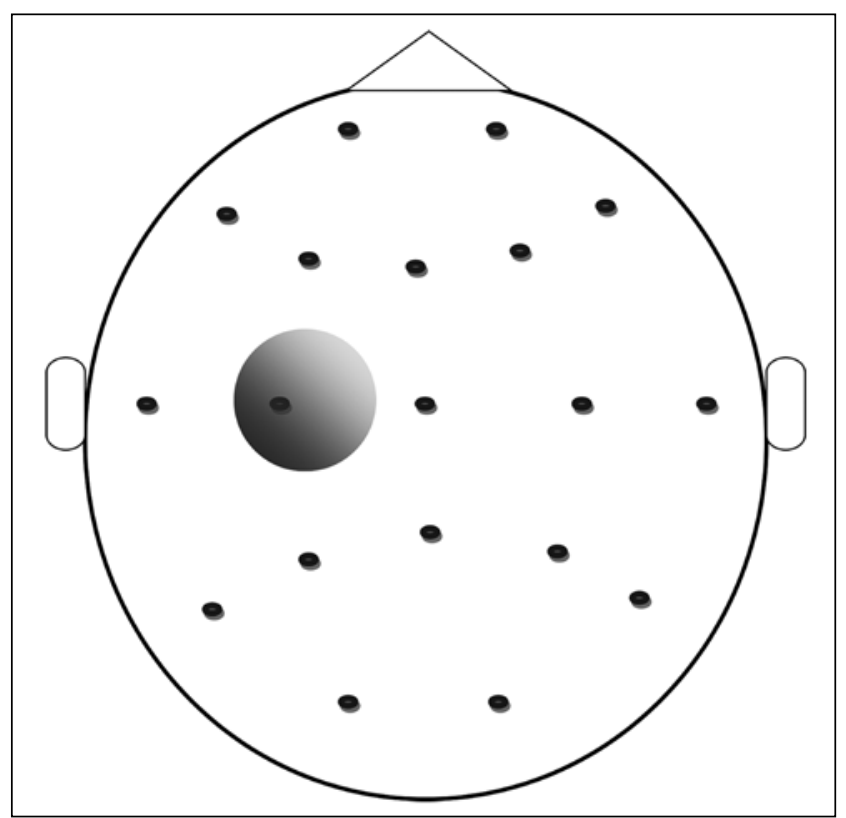

Figure 2. The epilepsy spectrum disorder (ESD) group has significantly higher beta2 power in the left-side central region than did the non-ESD group and the normal group (effect size, 0.370 $)^{20}$

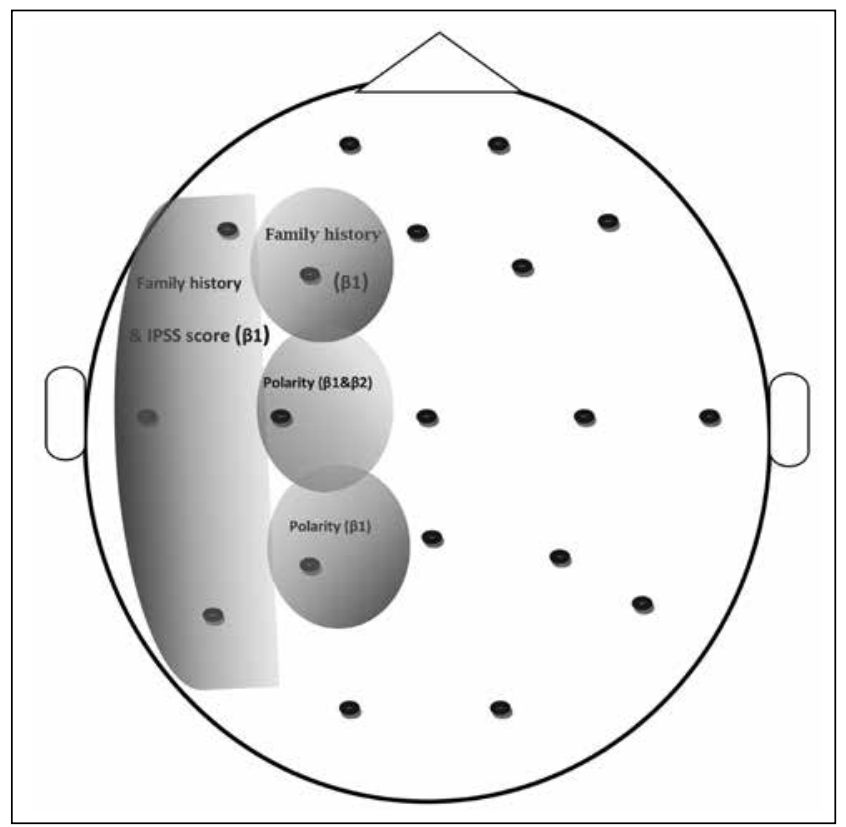

Figure 3. Correlation (point bi-serial) between clinicodemographic variables and left-side spectral power (betal band) in patients with depression and epilepsy spectrum disorder. 
depressive episodes $(p=0.050)$ and negatively correlated with left temporal beta1 power $(p=0.007)$. Patients with ESD who had a family history of affective disorder tended to have higher beta1 power in left-side frontal and temporal regions. Patients with unipolar ESD tended to have higher beta 1 and beta 2 power in the left-side central region and only higher beta 1 power in the left-side parietal region. Brain insult correlated with BDI-II score $(\mathrm{p}=0.041)$; patients with ESD who had a history of brain insult were likely to have more severe depression. Brain insult accounted for $20 \%$ $\left[R^{2}=(0.449)^{2}=0.201\right]$ of variability in BDI-II score.

The three groups did not differ significantly in terms of inter-hemispheric (five regions) and intra-hemispheric (ten regions) coherence.

The best two predictors for IIPSS score were left temporal beta1 power $(\mathrm{R}=0.569, \mathrm{~B}=-3.299, \mathrm{p}=0.007)$ and total number of previous depressive episodes $(\mathrm{R}=$ $0.686, \mathrm{~B}=1.237, \mathrm{p}=0.039)$, with $32.4 \%(\mathrm{R} 2=0.324)$ and $14.6 \%\left(R^{2}=0.470\right)$ explained variance, respectively.

\section{Discussion}

In the present study, those with ESD were defined as having a score of $\geq 70$ in IIPSS $^{3}$ or as having at least seven of 35 partial seizure-like symptoms on IIPSS (after standardising) ${ }^{5}$ More patients had brain insults especially MTBI in the ESD group than in the other two groups ( $p<0.05$, two-tailed). Brain injury is a risk factor for partial seizure-like phenomena. ${ }^{15}$

QEEG is a direct measure of the electrical energies of the brain and network dynamics, which are disturbed after a traumatic brain injury. ${ }^{21}$ Power spectrum is regarded as an index of activation of the cortex. Rhythmical activity from $13-35 \mathrm{~Hz}$ (beta frequency band) is considered an index of level of cortical excitation. In vitro and modelling studies have defined beta $(13-35 \mathrm{~Hz})$ oscillations as inhibitionbased rhythms. ${ }^{22,23}$ The beta oscillation is believed to represent an activated state of the underlying neuronal network. Beta frequencies reflect neuronal activation with gamma-aminobutyric acid (GABA) receptor action as pacemakers. ${ }^{24}$

Similar to patients with posttraumatic stress disorder, patients with ESD have an increase in beta activity, which can be due to global cortical hyperexcitability, prolonged wakefulness, or attention disturbances. ${ }^{25,26}$ An increase in beta activity can result from anxiety and restlessness, which are common clinical features in our patients with ESD. ${ }^{27}$ Patients with ESD had higher beta 1 and 2 activities in left-side temporal and central regions. Kindling has a role in epileptic-like phenomena secondary to repeated brain insult. $13,28,29$ This can be associated with local cortical excitability and/or complex partial seizure-like symptoms. Increased power in higher frequency bands indicates cortical hyperactivation, which is indicated by either increased lowfrequency band power or decreased high-frequency band power. Our QEEG findings (increased power in higher frequency bands such as beta) in patients with ESD differed from those reported in patients with MTBI involving cortical grey matter. ${ }^{21}$ Thus, we postulate that our patients with ESD probably did not have cortical grey matter injury.

At the cellular level, those with ESD are reported to have lowered GABAergic neurons density in deep hippocampal white matter and thus are more susceptible to hypoxia after anoxic spells or brain injuries. ${ }^{30}$ GABA receptors are involved in disequilibrium of beta frequency power. ${ }^{31}$ Vulnerability for ESD can be due to an increased excitatory drive and a deficient inhibitory tone, as evidenced in an alcoholism model. ${ }^{32}$ In our patients with ESD, complex partial seizure-like symptoms were correlated with the total number of depressive episodes. Increase in depressive episodes has a toxic effect on hippocampal cells and volume. ${ }^{33}$ Increase in the numbers of MTBI episodes and severe depressive episodes along with past depressive episodes makes depressive patients vulnerable to development of ESD.

Patients with unipolar ESD have elevated beta power in the right temporal region, whereas patients with bipolar ESD have elevated beta power in the left temporal region. ${ }^{34}$ In our study, patients with ESD who had a family history of affective disorder (especially mania) had higher beta1 power in left frontal and temporal regions, but patients with unipolar ESD had higher beta power in the left central and parietal regions, probably because of having additional MTBI.

We did not find changes in EEG coherence or EEG phase delays in the interhemispheric or intrahemispheric set. Changes in EEG coherence and EEG phase delays are usually linearly related to the magnitude of injury to both the grey matter and the white matter. ${ }^{21}$ Thus, coherence analysis did not support the model of hippocampal/limbic dysfunction in explaining the phenomenon of ESD.

In a pilot study of topographic brain mapping of EEG and evoked potential in Chinese subjects, normal subjects can be differentiated from patients with neuropsychiatric diseases such as Huntington disease, complex partial seizure, catatonic schizophrenic, and paranoid schizophrenic..$^{35}$

\section{Conclusion}

Some patients with depression may have a set of neurobehavioral disorders characterised by the subjective experience of multiple cognitive, affective, and psychosensory phenomena. In patients with severe depression who have multiple depressive episodes, clinicians should systematically check for episodic partial seizure-like phenomena, especially when patients have increased beta power and prior MTBI. Elevation in left-sided beta power is a marker/predictor for the risk of developing ESD and for treatment with anticonvulsant mood stabilisers.

\section{Acknowledgements}

We thank Dr Devosri Sen and Ms Swarnrekha Biswas for helping in manuscript writing, Dr Mohammad Zia Ul Haq 
and Mr Bipin Lal for helping in QEEG acquisition and data processing, and Dr B Das, Director, Central Institute of Psychiatry, Ranchi, India for helping in making the protocol.

\section{Declaration}

No funding received for this work from any of the organizations or so. There is no commercial or proprietary interest in any device, or equipment mentioned in the submitted article. There is no financial interest any author might have (as a consultant, stock owner, employee, evaluator, etc.) in any item mentioned in the article. All authors reported no biomedical financial interests or potential conflicts of interest.

\section{References}

1. Springer JA, Garvey MJ, Varney NR, Roberts RJ. Dichotic listening failure in dysphoric neuropsychiatric patients who endorse multiple seizure-like symptoms. J Nerv Ment Dis 1991;179:459-67. Crossref

2. Roberts RJ, Franzen K, Varney NR. Theta bursts, closed head injury, and partial seizure-like symptoms: a retrospective study. Appl Neuropsychol 2001;8:140-7. Crossref

3. Bob P, Susta M, Pavlat J, Hynek K, Raboch J. Depression, traumatic dissociation and epileptic-like phenomena. Neuro Endocrinol Lett 2005;26:321-5.

4. Bob P, Glaslova K, Susta M, Jasova D, Raboch J. Traumatic dissociation, epileptic-like phenomena, and schizophrenia. Neuro Endocrinol Lett 2006;27:321-6.

5. Roberts RJ, Gorman LL, Lee GP, Hines ME, Richardson ED, Riggle $\mathrm{TA}$, et al. The phenomenology of multiple partial seizure-like symptoms without stereotyped spells: an epilepsy spectrum disorder? Epilepsy Res 1992;13:167-77. Crossref

6. Varney NR, Hines ME, Bailey C, Roberts RJ. Neuropsychiatric correlates of theta bursts in patients with closed head injury. Brain Inj 1992;6:499-508. Crossref

7. Cope N. An integration of psychopharmacological and rehabilitation approaches traumatic brain injury rehabilitation. J Head Trauma Rehabil 1994;9:1-18. Crossref

8. Tierney JG, Fogel BS. Neuropsychiatric sequelae of mild traumatic brain injury. In: A. Stoudemire A, Fogel BS, editors. MedicalPsychiatric Practice. Volume 3. American Psychiatric Press; 1995.

9. Bob P. Dissociation and neuroscience: history and new perspectives. Int J Neurosci 2003;113:903-14. Crossref

10. Mesulam MM. Dissociative states with abnormal temporal lobe EEG. Multiple personality and the illusion of possession. Arch Neurol 1981;38:176-81. Crossref

11. Teicher MH, Glod CA, Surrey J, Swett C Jr. Early childhood abuse and limbic system ratings in adult psychiatric outpatients. J Neuropsychiatry Clin Neurosci 1993;5:301-6. Crossref

12. Teicher M, Andersen SL, Polcari A, Anderson CM, Navalta CP, Kim DM. The neurobiological consequences of early stress and childhood maltreatment. Neurosci Biobehav Rev 2003;27:33-44. Crossref

13. Rush AJ. Mood Disorder: Treatment of Depression. In: Sadock BJ, Sadock VA, editors. Kaplan and Sadock's Compressive Textbook of Psychiatry. Volume 1, 8th Ed. Lippincott Williams \& Wilkins; 2006.

14. Neppe VM, Kaplan C. Short-term treatment of atypical spells with carbamazepine. Clin Neuropharmacol 1988;11:287-9. Crossref

15. Roberts MA, Verduyn WH, Manshadi FF, Hines ME. Episodic symptoms in dysfunctioning children and adolescents following mild and severe traumatic brain injury. Brain Inj 1996;10:739-47. Crossref
16. World Health Organization. The ICD-10 Classification of Mental and Behavioral Disorders: Diagnostic Criteria for Research. 1992.

17. Practice parameter: the management of concussion in sports (summary statement). Report of the Quality Standards Subcommittee. Neurology 1997;48:581-5. Crossref

18. Jasper HH. The ten-twenty electrode system of the International Federation. Electroencephalogr Clin Neurophysiol 1958;10:371-5.

19. Newton TF, Leuchter AF, Walter DO, van Gorp WG, Morgenstern $\mathrm{H}$, Miller EN, et al. Electroencephalographic coherence in acquired immune deficiency syndrome. Psychiatry Res 1994;54;1-11. Crossref

20. Cohen J. A power primer. Psychol Bull 1992;112:155-9. Crossref

21. Thatcher RW. Electroencephalography and Mild Traumatic Brain Injury. In: Slobounov S, Sebastianelli W, editors. Foundations of Sport-Related Brain Injuries. Springer; 2006.

22. Haenschel C, Baldeweg T, Croft RJ, Whittington M, Gruzelier J. Gamma and beta frequency oscillations in response to novel auditory stimuli: a comparison of human electroencephalogram (EEG) data with in vitro models. Proc Natl Acad Sci U S A 2000;97:764550. Crossref

23. Whittington MA, Faulkner HJ, Doheny HC, Traub RD. Neuronal fast oscillations as a target site for psychoactive drugs. Pharmacol Ther 2000;86:171-90. Crossref

24. Whittington MA, Traub RD, Kopell N, Ermentrout B, Buhl EH. Inhibition-based rhythms: experimental and mathematical observations on network dynamics. Int J Psychophysiol 2000;38:315-36. Crossref

25. Niedermeyer E, DaSilva FL. Electroencephalography, Basic Principles. In: Niedermeyer E, DaSilva L, Editors. Clinical Applications and Related Fields, 3rd Ed. Williams and Wilkins; 1993.

26. Casada JH, Amdur R, Larsen R, Liberzon I. Psychophysiologic responsivity in posttraumatic stress disorder: generalized hyperresponsiveness versus trauma specificity. Biol Psychiatry 1998;44:1037-44. Crossref

27. Begic D, Hotujac L, Jokic-Begic N. Electroencephalographic comparison of veterans with combat-related post-traumatic stress disorder and healthy subjects. Int J Psychophysiol 2001;40:16772. Crossref

28. Putnam F. Dissociation in Children and adolescents. A developmental Perspective. The Guilford Press; 1997.

29. Post RM, Weis SR, Smith MA. Sensitization and Kindling. In: Friedman MJ, Charney DS, Deutch AY, editors. Neurobiological and Clinical Consequences of Stress: From Normal Adaptation to Posttraumatic Stress Disorder. Lippincott-Raven; 1995.

30. Ribak CE, Bradburne MR, Harris AB. A preferential loss of GABAergic, symmetric synapses in epileptic foci: a quantitative ultrastructural analysis of monkey neocortex. J Neurosci 1982;2:172535. Crossref

31. Porjesz B, Almasy L, Edenberg HJ, Wang K, Chorlian DB, Foroud T, et al. Linkage disequilibrium between the beta frequency of the human EEG and a GABAA receptor gene locus. Proc Natl Acad Sci U S A 2002;99:3729-33. Crossref

32. Begleiter H, Porjesz B. What is inherited in the predisposition toward alcoholism? A proposed model. Alcohol Clin Exp Res 1999;23:112535. Crossref

33. Gorwood P, Corruble E, Falissard B, Goodwin GM. Toxic effects of depression on brain function: impairment of delayed recall and the cumulative length of depressive disorder in a large sample of depressed outpatients. Am J Psychiatry 2008;165:731-9. Crossref

34. Flor-Henry P, Koles ZI, Howarth BC, et al. Neurophysiological Studies of Schizophrenia, Mania and Depression. In: Gruzelier J, Flor-Henry P, editors. Hemisphere Asymmetries of Function in Psychopathology. Elsevier; 1979.

35. Wong MTH, Lieh-Mak F. Topographic brain mapping of EEG and evoked potentials in Chinese normals and psychiatric patients: preliminary findings. J Hong Kong Coll Psychiatry 1991;1:6-11. 